\title{
The effects of a novel high intensity exercise intervention on established markers of cardiovascular disease and health in Scottish adolescent youth
}

\author{
Duncan S. Buchan, ${ }^{1}$ John D. Young, ${ }^{1}$ Alan D. Simpson, ${ }^{1}$ Non E. Thomas, ${ }^{2}$ \\ Stephen-Mark Cooper, ${ }^{3}$ Julien S. Baker ${ }^{1}$ \\ 'Institute of Clinical Exercise Science and Health, University of the West of Scotland, Hamilton, \\ Scotland; ${ }^{2}$ School of Human Sciences, Swansea University, Swansea, Wales; ${ }^{3}$ Cardiff School \\ of Sport, UWIC, Cyncoed Campus, Cardiff, Wales, UK
}

Significance for public health

Physical Education within schools is recognized as a site for promoting healthy behaviour patterns and for intervention. When you consider that in Scotland face-to-face contact time of Physical Education lessons can be less than $45 \mathrm{~min}$, those working with youth need to develop interventions that are not labour or time intensive, not expensive, and not difficult to implement, but which are modifiable, and can provide valid and reliable measurements. At the same time, the intervention should be capable of improving indices of health and well-being. In this study, we have devised a simple yet modifiable exercise intervention that has demonstrated significant improvements in indices of physical fitness. Thus, incorporating a high intensity exercise protocol into the Physical Education curriculum may prove useful in improving the health status of youth.

\begin{abstract}
This study examined the effects of high intensity exercise on physical fitness components and cardiovascular disease (CVD) risk factors in youth. Forty-one participants (15-17 years) were divided into a control and an intervention (high intensity exercise, HIT) group. The HIT group (15 boys, 2 girls) performed three weekly sessions over seven weeks consisting of either four to six repeats of maximal sprint running with $20-30 \mathrm{~s}$ recovery. The control group (20 boys, 4 girls) continued their normal activity patterns. All participants had indices of obesity and blood pressure (BP) recorded in addition to four physical performance measures pre-and post-intervention: cardiorespiratory fitness, muscular power, sprint speed and agility. In the HIT group, significant improvements in cardiorespiratory fitness $(\mathrm{P}<0.01)$ and agility $(\mathrm{P}<0.05)$ were noted. Participants in the control group, meanwhile, experienced a significant decrease in counter movement jump performance. These findings demonstrate that brief, intense exercise interventions are useful for improving indices of physical fitness in a short period of time.
\end{abstract}

\section{Introduction}

Obesity is a major risk factor in the aetiology of cardiovascular and metabolic diseases. Numerous risk factors, including poor dietary habits, physical inactivity, low aerobic fitness, obesity, being overweight and hypertension have all been linked to the development of cardiovascular disease (CVD). ${ }^{1}$ Although there is an extremely low risk of early mortality in youth, early and continued exposure to an unhealthy CVD profile may accentuate health issues. While the aetiology of CVD is complex, it is well established that engagement in a physically active lifestyle plays a crucial role in the prevention of current and future cardiovascular and metabolic diseases. ${ }^{2}$ Though regular participation in physical activity protects against future CVD risk, recent estimates suggest that current activity levels in youth are insufficient to meet recommendations. ${ }^{3}$ Childhood and adolescence have been identified as critical periods for developing favourable activity habits. These habits may continue into adulthood, and any lifestyle interventions that influence this modifiable contributor to future CVD risk are desirable.

Physical Education (PE) programmes within schools is an ideal setting to implement lifestyle interventions, and is crucial for the promotion of the health and well-being of youth. However, activity levels in PE tend to vary considerably within and between classes, as a result of pedagogical, individual, and environmental factors. ${ }^{4}$ Recent evidence suggests that youth spend less than $50 \%$ of PE time in moderate intensity activity. ${ }^{3}$ From their review, Fairclough and Stratton found that in classes taught under non-intervention conditions, students engaged in moderate intensity activity for $34.2 \%$ of available time, regardless of the measurement instrument used.

As PE may be the only meaningful opportunity for some to engage in physical exercise, it is important that the stimulus afforded to them is appropriate to improve their health and well being. As the appropriateness of current youth physical activity (PA) guidelines have been questioned,${ }^{5}$ there may be a need to consider the potential of non-traditional exercise interventions as a method of improving the health and wellbeing of youth.

Previous investigations that have examined the effects of high intensity exercise (HIT) upon CVD risk factors have consistently supported the role of HIT as a means of improving health and well-being. ${ }^{6-9}$ Though these studies all involved adult participants, the benefits of such an approach is not restricted to adults. Superior benefits of an aerobic interval training (AIT) intervention lasting only three months were found in overweight and obese adolescents in comparison to a multidisciplinary approach that involved exercise, nutritional and psychological advice, twice a month for 12 months. ${ }^{10}$ From the first 3-month follow-up session, participants involved in the AIT group experienced significantly greater improvements in cardiorespiratory fitness, endothelial function, measures of adiposity and blood pressure, and in resting measures of fasting glucose and high-density lipoprotein cholesterol (HDLC) than those involved in the multidisciplinary approach.

Though these studies have demonstrated the potency of HIT as a means of improving CVD risk factors, when you consider the exercise time commitment of each session in the following studies (38-40 $\min ), 6,7,10$ it is still unclear whether HIT of a short duration would still impact upon the health and well-being of individuals. In addition, one 
has to query whether individuals that do not have poor CVD risk profiles and are of a healthy weight would be suitably motivated to participate and adhere to such a vigorous exercise intervention.

The purpose of this study, therefore, was to determine the effects of an HIT intervention on components of physical fitness, namely: cardiorespiratory performance, muscular power and speed/agility. ${ }^{1} \mathrm{~A}$ second aim was to investigate the effects of the intervention on traditional markers of CVD: blood pressure, percentage body fat (\%BF) and body mass index (BMI).

\section{Design and Methods}

\section{Subjects}

Forty-one youths (35 boys and 6 girls aged 15-17 years) volunteered to participate in the study. Ethical approval was obtained from the University of the West of Scotland Ethics Committee. Participants were recruited from two PE classes in years 5 and 6 . Subjects were randomly assigned to a non-exercising control group or to a HIT group.

\section{Anthropometry and physical fitness measures}

Details of anthropometric and blood pressure measures have been published previously. ${ }^{11}$ Three components of health-related physical fitness were measured following three familiarization periods. Cardiorespiratory fitness was measured using the 20 m multistage fitness test (MSFT). ${ }^{12}$ The counter movement jump (CMJ) was used to measure muscular power. Jumping height was measured with the Optojump system (Microgate, Bolzano, Italy). Running speed was measured over $10 \mathrm{~m}$ using an electronic sprint timer with photoelectric sensors (Polifemo Radio Light, Microgate). Agility was measured with the 505 test using two photoelectric sensors (Polifemo Radio Light, Microgate) placed $5 \mathrm{~m}$ from the starting line and $5 \mathrm{~m}$ from a designated turning point. All measurements were taken pre-and post-intervention following standard procedures and a standardized warm-up. ${ }^{11}$ All of the post-intervention testing was performed within five days of completion of the last exercise session.

\section{Heart rate and physical activity}

Heart rate (HR) response was recorded on participants once during each training week with continuous heart rate telemetry (Hosand, TM200, Hosand Technology, Verbania, Italy). All participants completed a validated physical activity questionnaire for adolescents (PAQ-A), ${ }^{13}$ which required them to recall their PA over the previous seven days.

\section{Intervention protocol}

Participants in the HIT group (15 boys, 2 girls) were required to complete a $30 \mathrm{~s}$ maximal effort sprint within a $20 \mathrm{~m}$ distance separated by cones. This equated to 1 repetition. Training progression was implemented by increasing the number of repetitions from four during weeks 1 and 2 , to five during weeks 3 and 4 , and to six during weeks 5 and 6 . During weeks 1-6, each repetition was interspersed by $30 \mathrm{~s}$ intervals. During week 7 , participants still performed six repetitions but each was interspersed by only $20 \mathrm{~s}$ recovery. The protocol was performed 3 times weekly and was performed at the start of every PE class. The control group ( 20 boys, 4 girls) were instructed to maintain normal activity patterns throughout the intervention period.

\section{Data analysis}

Means and standard deviations were calculated for each observation point. The level of significance was set at $\mathrm{P} \leq 0.05$ throughout. The Anderson-Darling test was applied to confirm normality, while homogeneity of variance was assessed using Levene's test. The results were analyzed using a two way repeated measure analysis of variance with one between subjects variable (Groups: Control and HIT) and one within subjects variable (Time: Pre and Post). Where a Group $\times$ Time interaction was significant $(\mathrm{P}<0.05)$ the simple effects of Time were investigated for both the Control and HIT groups. These simple effects were examined either using separate one-way repeated measurement analysis or, where the analysis of the residuals suggested non-normality, using the non parametric Wilcoxon's signed ranks test.

\section{Results}

Descriptive statistics and the effects of the intervention on physical and physiological measures are displayed in Table 1. Post intervention measures revealed that there was a significant decrease $(\mathrm{P}<0.05)$ in 20

Table 1. Descriptive statistics with pre- and post-measures of physical and physiological variables. Data are presented as mean \pm SD.

\begin{tabular}{|c|c|c|c|c|c|c|c|}
\hline \multirow[b]{2}{*}{ Physical } & \multicolumn{2}{|c|}{ Control group } & \multicolumn{2}{|c|}{ HIT group } & \multicolumn{2}{|c|}{ Main effects } & \multirow{2}{*}{$\begin{array}{l}\text { Interaction } \\
\text { Group } \times \text { time }\end{array}$} \\
\hline & Pre & Post & Pre & Post & Group & Time & \\
\hline Stature (cm) & $171.1 \pm 8.7$ & $172.4 \pm 8.9$ & $171.1 \pm 7.9$ & $172.6 \pm 7.5$ & 0.488 & 0.000 & 0.488 \\
\hline Body mass (kg) & $66.2 \pm 7.5$ & $66.4 \pm 7.9$ & $63.4 \pm 9.2$ & $63.7 \pm 9.3$ & 0.300 & 0.333 & 0.714 \\
\hline BMI $\left(\mathrm{kg} / \mathrm{m}^{2}\right)$ & $22.7 \pm 2.6$ & $22.4 \pm 2.5$ & $21.6 \pm 2.2$ & $21.3 \pm 2.1$ & 0.154 & 0.001 & 0.786 \\
\hline WHR & $0.8 \pm 0.0$ & $0.8 \pm 0.0$ & $0.8 \pm 0.1$ & $0.8 \pm 0.1$ & 0.921 & 0.009 & 0.682 \\
\hline Body fat (\%) & $18.1 \pm 8.9$ & $17.2 \pm 7.7$ & $16.9 \pm 5.1$ & $17.2 \pm 3.6$ & 0.801 & 0.541 & 0.203 \\
\hline \multicolumn{8}{|l|}{ Physiological } \\
\hline $\mathrm{CMJ}(\mathrm{cm})$ & $32.5 \pm 7.1(23)^{\circ}$ & $30.22 \pm 5.1(23)^{\circ}$ & $33.1 \pm 4.3$ & $34.3 \pm 6.1$ & 0.203 & 0.314 & 0.003 \\
\hline 10m sprint (s) & $2.0 \pm 0.2$ & $2.0 \pm 0.2$ & $2.0 \pm 0.2$ & $1.9 \pm 0.2$ & 0.082 & 0.070 & 0.064 \\
\hline SBP (mm Hg) & $113 \pm 10$ & $109 \pm 11$ & $112 \pm 10$ & $106 \pm 11$ & 0.496 & 0.002 & 0.571 \\
\hline DBP (mm Hg) & $68 \pm 8$ & $64 \pm 7$ & $67 \pm 7$ & $65 \pm 6$ & 0.900 & 0.001 & 0.186 \\
\hline Aerobic fitness (shuttles) & $81.3 \pm 25.3$ & $80.1 \pm 24.7$ & $82.0 \pm 25.8$ & $88.8 \pm 26.4$ & 0.345 & 0.081 & 0.002 \\
\hline 505 agility test (s) & $2.5 \pm 0.2$ & $2.6 \pm 0.3$ & $2.6 \pm 0.3$ & $2.5 \pm 0.2$ & 0.361 & 0.224 & 0.022 \\
\hline
\end{tabular}

HIT, high intensity exercise; BMI, body mass index; WHR, waist-to-hip ratio; CMJ, counter movement jump; SBP, systolic blood pressure; DBP, diastolic blood pressure. ${ }^{\circ}$ Where $n \neq$ denoted number, actual sample number is presented in brackets. 
Table 2. Mean differences (95\% confidence intervals) for variables that differed significantly in the post-hoc analyses.

\begin{tabular}{|c|c|c|c|c|}
\hline Variable & Control group & $\mathbf{P}$ & HIT group & $\mathbf{P}$ \\
\hline CMJ (cm) & Pre vs Post 2.30 [0.89106 - 3.71764] & 0.003 & & \\
\hline Aerobic fitness ( shuttles) & & & Pre vs Post -7.06 [-10.77298 - -3.34467] & 0.001 \\
\hline 505 agility test (s) & & & Pre vs Post .01965 [0.01965 - 0.18035] & 0.018 \\
\hline
\end{tabular}

HIT, high intensity exercise; CMJ, counter movement jump.

Table 3. Mean heart rate response recorded during each week. Data are presented as mean $\pm S D$.

High intensity group
\[ \mathrm{n}=17 \]
Heart rate response $(\mathrm{bpm})$

\begin{tabular}{ll} 
Week 1 & $177.9 \pm 14.9(11)^{\circ}$ \\
Week 2 & $178.2 \pm 13.4(10)^{\circ}$ \\
\hline Week 3 & $174.8 \pm 15.5(11)^{\circ}$ \\
Week 4 & $178.2 \pm 16.5(12)^{\circ}$ \\
\hline Week 5 & $177.7 \pm 18.0(12)^{\circ}$ \\
Week 6 & $175.1 \pm 18.9(11)^{\circ}$ \\
\hline Week 7 & $174.3 \pm 19.4(10)^{\circ}$ \\
\hline${ }^{\circ}$ Where $n \neq$ denoted number, actual sample number is presented in brackets.
\end{tabular}

MSFT and agility performance for the participants in the HIT group (Table 2). Participants in the control group also experienced a significant decrease in CMJ performance post-intervention. Mean PA levels estimated using the PAQ-A were $2.0 \pm 0.8$ (control group) and $2.2 \pm 0.4$ (HIT group). There was no significant difference in activity levels within or between groups either pre- or post-intervention. Mean heart rate response in the HIT group ranged from $174.3 \pm 19.4$ to $178.2 \pm 16.5 \mathrm{bpm}$ during the intervention period (Table 3 ).

\section{Discussion}

The exercise time commitment for the HIT group over the seven weeks was only 54 min with no injuries reported during this period. The main findings from this study indicated that brief high intensity exercise over a short intervention period (3 sessions per week for 7 weeks); can improve components of physical fitness; namely; cardiorespiratory fitness and agility. Physical fitness is considered to be an important health marker, as well as a predictor of morbidity and mortality for cardiovascular disease. ${ }^{5}$ Given the time constraints of school curricula, incorporating a HIT protocol into the PE curriculum may function to improve the PA levels and the health status of youth. Further development of intense exercise interventions that can be implemented within the school setting seems warranted.

\section{References}

1. Ortega FB, Ruiz JR, Castillo MJ, Sjostrom M. Physical fitness in childhood and adolescence: a powerful marker of health. Int J Obes 2008;32:1-11.

2. Strong WB, Malina RM, Blimkie CJ, et al. Evidence based physical activity for school-age youth. J Pediatr 2005;146:732-7.

3. Fairclough SJ, Stratton G. A review of physical activity levels during elementary school physical education. J Teach Phys Educ 2006;25:
Correspondence: Dr. Duncan S. Buchan, Institute of Clinical Exercise Science and Health, University of the West of Scotland, Hamilton, ML3 0JB, Scotland, UK. Tel. +44.01698 .283100 .

E-mail: duncan.buchan@uws.ac.uk

Key words: health, youth, high intensity exercise.

Acknowledgments: the authors gratefully acknowledge Ms. Maini Tulokas in the Social Insurance Institution for her skilled help in preparing the figures. Funding: this study was financially supported by the Chief Scientist Office for Scotland.

Contributions: DSB, data responsibility and manuscript writing; SMC, NET,

JY, AS, JSB critical comments on the manuscript.

Conflict of interests: the authors report no conflict of interest.

Received for publication: 30 November 2011.

Accepted for publication: 30 March 2012.

CCopyright D.S. Buchan et al., 2012

Licensee PAGEPress, Italy

Journal of Public Health Research 2012; 1:e24

doi:10.4081/jphr.2012.e24

This work is licensed under a Creative Commons Attribution NonCommercial 3.0 License (CC BY-NC 3.0).

\section{9-57.}

4. Stratton G. Children's heart rates during physical education lessons: A review. Pediatr Exerc Sci 1996;8:215-33.

5. Andersen LB, Harro M, Sardinha LB, et al. Physical activity and clustered cardiovascular risk in children: a cross-sectional study (The European Youth Heart Study). Lancet 2006;368:299-304.

6. Wisloff U, Stoylen A, Loennechen JP, et al. Superior cardiovascular effect of aerobic interval training versus moderate continuous training in heart failure patients: a randomized study. Circulation 2007;115:3086-94.

7. Tjonna AE, Lee SJ, Rognmo 0, et al. Aerobic interval training versus continuous moderate exercise as a treatment for the metabolic syndrome: a pilot study. Circulation 2008;118:346-54.

8. Burgomaster KA, Heigenhauser GJ, Gibala MJ. Effect of short-term sprint interval training on human skeletal muscle carbohydrate metabolism during exercise and time-trial performance. J Appl Physiol 2006;100:2041-7.

9. Burgomaster KA, Hughes SC, Heigenhauser GJ, et al. Six sessions of sprint interval training increases muscle oxidative potential and cycle endurance capacity in humans. J Appl Physiol 2005;98:1985-90.

10. Tjonna AE, Stolen TO, Bye A, et al. Aerobic interval training reduces cardiovascular risk factors more than a multitreatment approach in overweight adolescents. Clin Sci 2009;116:317-26.

11. Buchan DS, Ollis S, Thomas NE, Baker JS. The influence of a high intensity physical activity intervention on a selection of health related outcomes: an ecological approach. BMC Public Health 2010;10:8.

12. Leger LA, Mercier D, Gadoury C, Lambert J. The multistage 20 metre shuttle run test for aerobic fitness. J Sports Sci 1988;6:93-101.

13. Kowalski KC, Crocker PRE, Kowalski NP. Convergent validity of the physical activity questionnaire for adolescents. Pediatr Exerc Sci 1997:342-52. 